\title{
Application of a Real Gas Model by van der Waals for a Hydrogen Tank Filling Process
}

\author{
Maximilian Kormann $^{1} \quad$ Imke Lisa Krüger $^{2}$ \\ ${ }^{1}$ Dassault Systèmes, Germany, maximilian.kormann@3ds.com \\ ${ }^{2}$ Dassault Systèmes, Germany, imkelisa.krueger@3ds. com
}

\begin{abstract}
Hydrogen fuel tanks operate at high system pressure levels. In these regions effects occur, which cannot be handled by an ideal gas model. One of these is the JouleThomson effect. It describes an adiabatic throttling without change in enthalpy, but a change in temperature. The tank filling process can be simplified to a throttling valve, so the effect is of interest. In this investigation the van der Waals equations are implemented in a real gas model for the Hydrogen Library and the Pneumatic Systems Library $(P S L)$ by Dassault Systèmes and the model is applied to a hydrogen tank filling process. Performance and accuracy are compared to the CoolProp fluid properties library, which is imported with the ExternalMedia library.
\end{abstract}

Keywords: Hydrogen, Pneumatic Systems Library, Tank Filling Process, Medium Models, Real Gas, van der Waals, Joule-Thomson-Effect, CoolProp

\section{Introduction}

The calculation of thermodynamic properties plays an important role in simulating thermohydraulic systems. The specific behaviour we want to study as well as the pressure and temperature range define the exactness needed of the medium properties model. The ideal gas approach is only valid for low pressures, complex approaches that cover the liquid phase as well slow down the simulation speed.

The van der Waals model gives a simplified analytical approach and it assumes the deviation to be acceptable for e.g. early stages of design or if only the qualitative behavior should be shown. The Joule-Thomson effect that is to be examined in this paper can be analytically modeled and studied with the van der Waals approach. It results in simple equations for e.g. the internal pressure.

The model has been implemented for the Hydrogen and Pneumatic Systems Library by Dassault Systèmes. New gases can be easily added, the only parameters needed are the molar mass of the gas, the critical pressure and temperature, the sutherland constants and the table-based specific internal energy.

A recent application where this effect occurs is the hydrogen tank filling processes for fuel cell vehicles. Hydrogen has to be stored at high pressures e.g. 200 bar due to the low energy density at low pressures. In order to be competitive to conventionally fueled cars, tank filling should take less than $200 \mathrm{~s}$ for 5 kilogram (US DOE et al., 2009). For safety reasons, the temperature inside the tank may not exceed $85^{\circ} \mathrm{C}$.

Due to its small molecules, hydrogen shows a different behavior when compressed or expanded than e.g. air. When air is expanded isenthalpic, temperature sinks where as for hydrogen the temperature rises. This is due to the different signs of the Joule-Thomson coefficient, that describes the temperature evolution during a pressure change.

To design the tank filling processes and identify cooling needs during the injection, system simulation is the best way. We will demonstrate in this paper that the van der Waals approach can be used for hydrogen to evaluate tank filling processes at an early stage of development.

\section{Thermodynamic background}

To describe the temperature change at an adiabatic throttling some thermodynamic basics have to be given.

\subsection{Ideal Gas}

The ideal gas model assumes the fluid to consist of dimensionless particles which can move freely in the given volume until they hit another particle or the wall. The thermal equation of state between the pressure $p$ and temperature $T$ is defined according to (Adkins, 1983; Tschoegl, 1983) with the specific gas constant $R$ :

$$
R T=p v
$$

and the specific volume

$$
v=\frac{V}{m}=\frac{1}{\rho}
$$

\subsection{Real gas equation by van der Waals}

The ideal gas model lacks two effects: Interactive forces between the fluid molecules and their dimension. For the first effect, the pressure in the van der Waals model (der Waals, 1967) is increased by the internal pressure of the gas. The influence of the dimension of the molecules is considered by reducing the specific volume by the volume of the fluid molecules:

$$
R T=\left(p+\frac{a}{v^{2}}\right)(v-b)
$$


The two new parameters can be derived from the critical state of the fluid (Weigand et al., 2008). The internal pressure is defined using the cohension pressure parameter:

$$
a=\frac{27\left(R T_{c}\right)^{2}}{64 p_{c}}
$$

The volume occupied by the molecules can be written directly:

$$
b=\frac{R T_{c}}{8 p_{c}}
$$

\subsection{Internal Pressure}

The internal pressure of a fluid is defined as the partial derivation of the specific internal energy $u$ by the specific volume at constant temperature (Moore, 1986):

$$
\pi_{T}=\left(\frac{\delta u}{\delta v}\right)_{T}
$$

It can be derived from the fundamental thermodynamic relation with the specific entropy $s$ under assumption of a constant amount of mass (Callen, 1985) by deriving the internal energy partially by the volume at constant temperature:

$$
\begin{aligned}
d u & =T d s-p d v \\
\left(\frac{\delta u}{\delta v}\right)_{T} & =T\left(\frac{\delta s}{\delta v}\right)_{T}-p
\end{aligned}
$$

With the Maxwell-Relation (Weigand et al., 2008)

$$
\left(\frac{\delta s}{\delta v}\right)_{T}=\left(\frac{\delta p}{\delta T}\right)_{v}
$$

the expression for the internal pressure becomes:

$$
\pi_{T}=\left(\frac{\delta u}{\delta v}\right)_{T}=T\left(\frac{\delta p}{\delta T}\right)_{v}-p
$$

For an ideal gas we get from equation (1):

$$
\begin{aligned}
\left(\frac{\delta p}{\delta T}\right)_{v} & =\frac{R}{v} \\
\pi_{T} & =T\left(\frac{\delta p}{\delta T}\right)_{v}-p=T \frac{R}{v}-p=0
\end{aligned}
$$

As the ideal gas equation does not model forces between the fluid molecules except elastic collisions, the internal pressure is zero. Thus the Joule-Thomson effect cannot be covered by an ideal gas law. In the real gas equation the van der Waals forces between the molecules are covered by the internal pressure term. From equation (3) we get:

$$
\begin{aligned}
\left(\frac{\delta p}{\delta T}\right)_{v} & =\frac{R}{v-b} \\
\pi_{T} & =T\left(\frac{\delta p}{\delta T}\right)_{v}-p=T \frac{R}{v-b}-p \\
& =T \frac{R}{v-b}-\left(\frac{R T}{v-b}-\frac{a}{v^{2}}\right)=\frac{a}{v^{2}}
\end{aligned}
$$

The internal pressure for a real gas modeled with the van der Waals equation is always positive.

\subsection{Caloric equation of state}

Assuming a constant amount of mass the total differential of the internal energy is (Weigand et al., 2008):

$$
d u=\left(\frac{\delta u}{\delta T}\right)_{v} d T+\left(\frac{\delta u}{\delta v}\right)_{T} d v=c_{v} d T+\pi_{T} d v
$$

For an ideal gas, the internal pressure is zero and thus the internal energy only depends on the temperature with the specific heat capacity at constant volume $c_{v}$.

\subsection{Specific Enthalpy}

The enthalpy describes the energy of a system. The enthalpy can only change when energy is transferred over the system border. The specific enthalpy of a fluid is defined as (Weigand et al., 2008):

$$
h=u+p v
$$

For an ideal gas the expression simplifies to

$$
h=u+R T
$$

As described according to equation (16), for an ideal gas the internal energy only depends on the temperature. In this case, also the enthalpy only depends on the inner energy. This is not the case for a real gas.

\subsection{The Joule-Thomson effect}

When a gas is expanded from a high pressure over an isenthalpic valve, a change in temperature can be observed. This behaviour cannot be described by an ideal gas model as it does not model a pressure dependency of the specific enthalpy as mentioned in section 2.5. The effect is described by the Joule-Thomson coefficient (Greiner et al., 1993):

$$
\mu_{J T}=\left(\frac{\delta T}{\delta p}\right)_{h}
$$

To derive its equation, the total differential of the specific entropy under assumption of a constant amount of mass will be set into the fundamental thermodynamic relation from equation (7). It reduces the dependency of the change in enthalpy to pressure and temperature:

$$
\begin{aligned}
d s & =\left(\frac{\delta s}{\delta T}\right)_{p} d T+\left(\frac{\delta s}{\delta p}\right)_{T} d p \\
d h & =d u+p d v+v d p \\
d h & =T\left(\frac{\delta s}{\delta T}\right)_{p} d T+T\left(\frac{\delta s}{\delta p}\right)_{T} d p+V d p
\end{aligned}
$$


With the definition of the specific heat capacity at constant pressure $c_{p}$ and the Maxwell-Relation for the Gibbs energy we get:

$$
\begin{aligned}
T\left(\frac{\delta s}{\delta T}\right)_{p} & =c_{p} \\
\left(\frac{\delta s}{\delta p}\right)_{T} & =-\left(\frac{\delta v}{\delta T}\right)_{p} \\
d h & =c_{p} d T-T\left(\frac{\delta v}{\delta T}\right)_{p} d p+V d p
\end{aligned}
$$

Now we can set $d h=0$ and rearrange:

$$
\mu_{J T}=\left(\frac{\delta T}{\delta p}\right)_{H}=\frac{1}{c_{p}}\left(T\left(\frac{\delta v}{\delta T}\right)_{p}-v\right)
$$

For $v>>b$ and $v>>a / R T$ the real gas equation (3) can be rewritten and solved by $v$ (Greiner et al., 1993):

$$
\begin{aligned}
& p=\frac{R T}{v}\left(1+\frac{b}{v}-\frac{a}{v R T}+\frac{a b p}{v R^{2} T^{2}}\right) \\
& v=\frac{R T}{p}+b-\frac{a}{R T}+\frac{a b p}{R^{2} T^{2}}
\end{aligned}
$$

Then the inner differential from equation (26) can be formulated and put into the equation of the Joule-Thomson coefficient:

$$
\begin{aligned}
\left(\frac{\delta v}{\delta T}\right)_{p} & =\frac{R}{p}+\frac{a}{R T^{2}}-2 \frac{a b p}{R^{2} T^{3}} \\
\mu_{J T} & =\frac{1}{c_{p}}\left(\frac{2 a}{R T}-b-3 \frac{a b p}{R^{2} T^{2}}\right)
\end{aligned}
$$

\section{Implementation as a real gas model}

The equations from section 2.2 and 2.4 are introduced as an additional fluid model in the Hydrogen Library and the Pneumatic Systems Library (PSL). The dynamic viscosity is estimated by the law of (Sutherland, 1893).

\subsection{Density}

The density is estimated according to the real gas model by van der Waals from equation (3).

\subsection{Specific Enthalpy}

To get a non-differential formulation of the specific enthalpy, equation (16) has to be integrated from a reference point indicated by a subscript 0 and put into equation (17):

$$
\begin{aligned}
& h=\int_{T_{0}}^{\int^{T}} c_{v} d T+\int_{v_{0}}^{v} \pi_{T} d v+p v \\
& h=\underbrace{\int_{T_{0}}^{T} c_{v} d T-\frac{a}{v_{0}}}_{u_{T}(T)}+\frac{a}{v}+p v
\end{aligned}
$$

As there is no easy-to-handle analytical expression for $c_{v}$ and to avoid the use of polynomials, the temperaturedependent part of the specific internal energy and the internal pressure correction of the reference point (marked with an under-brace in equation (31)) is provided as a tableinterpolation $u_{T}(T)$. Then we get the following equation for the specific enthalpy:

$$
h=u_{T}(T)+p v+\frac{a}{v}
$$

\subsection{Parametrization}

Compared to ideal gas models, the additional parameters for creating a new gas model are pressure and temperature in the critical state as well as the tables for the specific internal energy and heat capacity (see table 1). As the specific heat capacity is described by a look-up-table, the degrees of freedom are no longer necessary for the van der Waals Real Gas Model.

Table 1. Required parameters for the gas models.

\begin{tabular}{lcc}
\hline Gas model & Ideal & Real \\
\hline Molar mass & $\bullet$ & $\bullet$ \\
Dynamic viscosity at ref. point & $\bullet$ & $\bullet$ \\
Viscosity ref. point temperature & $\bullet$ & $\bullet$ \\
Sutherland constant & $\bullet$ & $\bullet$ \\
Degrees of freedom & $\bullet$ & $\bullet$ \\
Critical pressure & & $\bullet$ \\
Critical temperature & & $\bullet$ \\
Temperature table & \\
Specific internal energy table & \\
Specific heat capacity table & & $\bullet$ \\
\hline
\end{tabular}

\section{Benchmark of the van der Waals model}

To determine a range for the validity of the gas model, it will be compared to generated values by the CoolProp library (Bell et al., 2014) for pure Hydrogen gas. The open source $\mathrm{C}++$ library CoolProp provides equations of state and transport properties for pure fluids and mixtures. The relative deviations of results for various fluid properties of hydrogen compared to the NIST Reference Fluid Thermodynamic and Transport Properties Database (Lemmon et al., 2010) are in the range of $1 \times 10^{-4}$ to $1 \times 10^{-14}$.

The CoolProp library is interfaced with the help of the ExternalMedia library (Casella and Richter, 2008). The ExternalMedia provides medium packages that can be used directly in the Pneumatic Systems Library and in the Hydrogen library.

\subsection{Density of hydrogen}

In the following, the influence of the gas model on the density will be outlined in the case of hydrogen. 


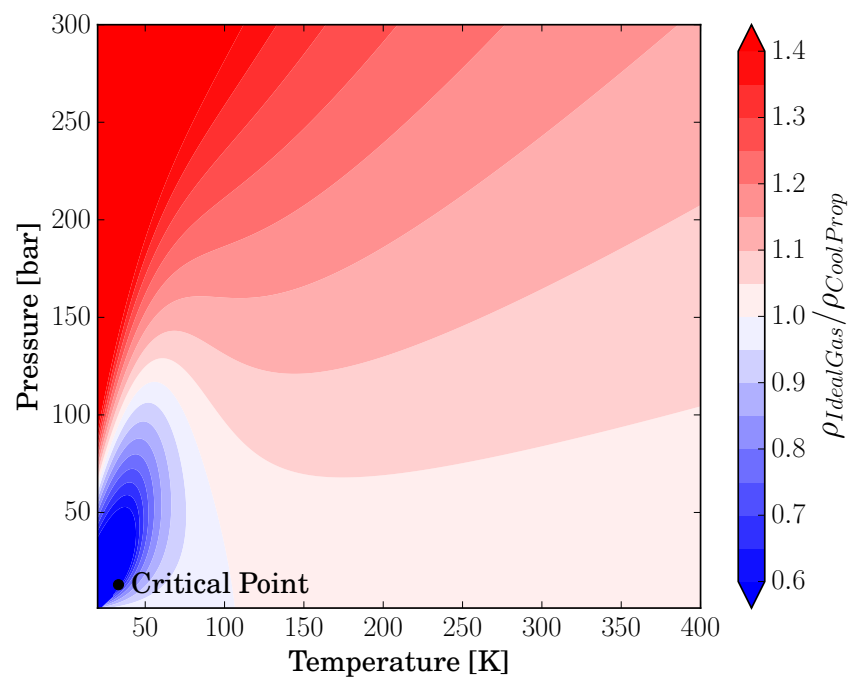

Figure 1. Comparison of the density between the ideal gas model and the CoolProp model for hydrogen.

The ideal gas model has a quite small range of validity: To keep the error in the density below $5 \%$, the pressure has to be below 50 bar and the temperature has to be above $100 \mathrm{~K}$ (see Figure 1). As discussed in section 2.3, the Joule-Thomson effect can not be covered by the ideal gas law, as it does not consider intermolecular forces.

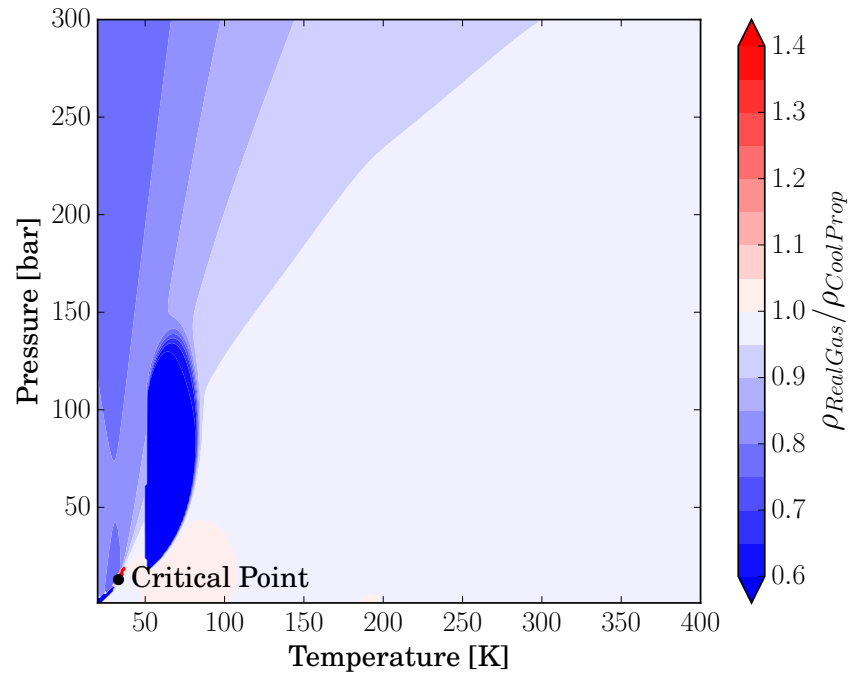

Figure 2. Comparison of the density between the van der Waals gas model and the CoolProp model for hydrogen.

The van der Waals model has a much wider range of validity (see Figure 2). Pressures up to 200 bar and temperatures above $100 \mathrm{~K}$ can be covered with an error in the density below $5 \%$. For the same pressure and temperature the error is smaller than for the ideal gas model. Besides the wider range of application, the Joule-Thomson effect can be covered by the van der Waals air model, as discussed in section 2.3.

\subsection{Density of air}

Though air is a mixture of several gases, it still can be modeled by the van der Waals real gas approach. As stated in section 2.5 , only the critical state of the fluid has to be known. This can be measured for air. Again, the density of the gas model will be compared to the CoolProp library.

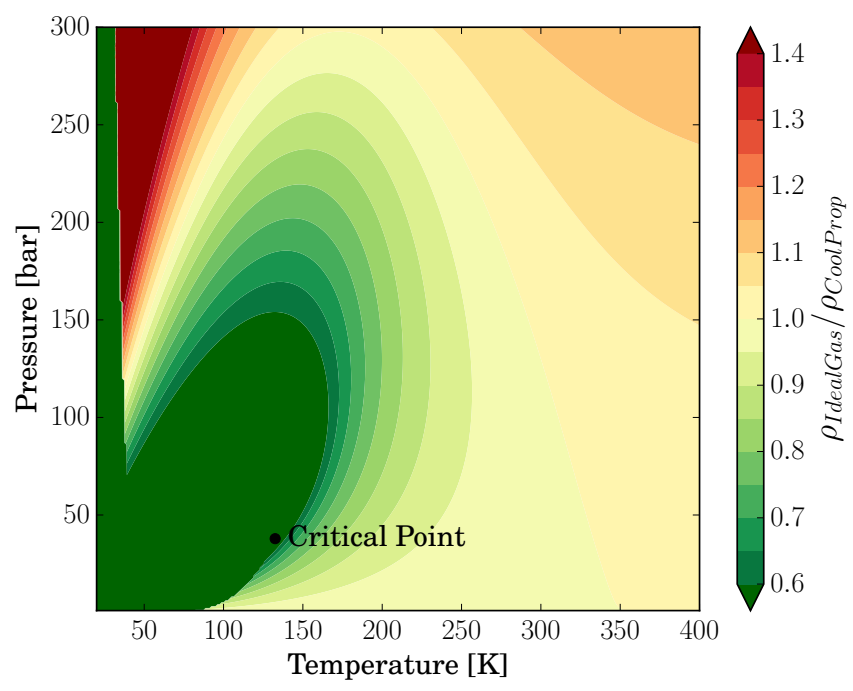

Figure 3. Comparison of the density between the ideal gas model and the CoolProp model for air.

The ideal gas model has quite small range of validity: To keep the error in the density below $5 \%$, the pressure has to be below 150 bar and the temperature has to be above $250 \mathrm{~K}$ (see Figure 3).

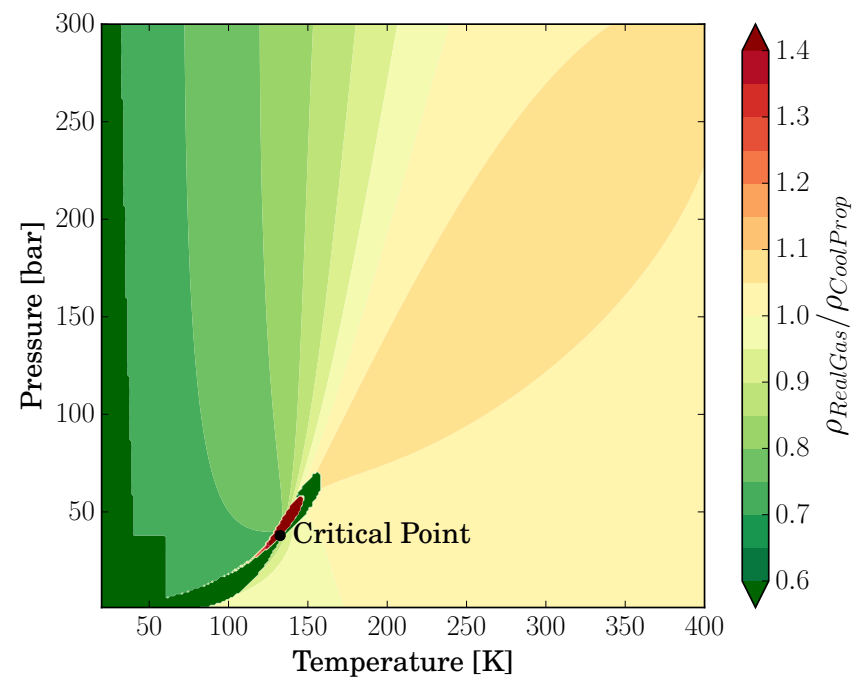

Figure 4. Comparison of the density between the van der Waals gas model and the CoolProp model for air.

The van der Waals air model has a slightly wider range of validity (see Figure 4). Pressures up to 200 bar and temperatures above $170 \mathrm{~K}$ can be covered with an error in the density below 5\%. The benefit in application range by using the van der Waals model for air is smaller than for 
hydrogen. Still low temperatures can be handled with a higher precision and the Joule-Thomson effect is covered.

\subsection{Joule-Thomson coefficient}

In section 2.6 an analytic formulation for the JouleThomson coefficient has been derived in equation (30). It will be compared to the direct derivative of the enthalpy calculated in the $P S L$ according to equation (19) for hydrogen:
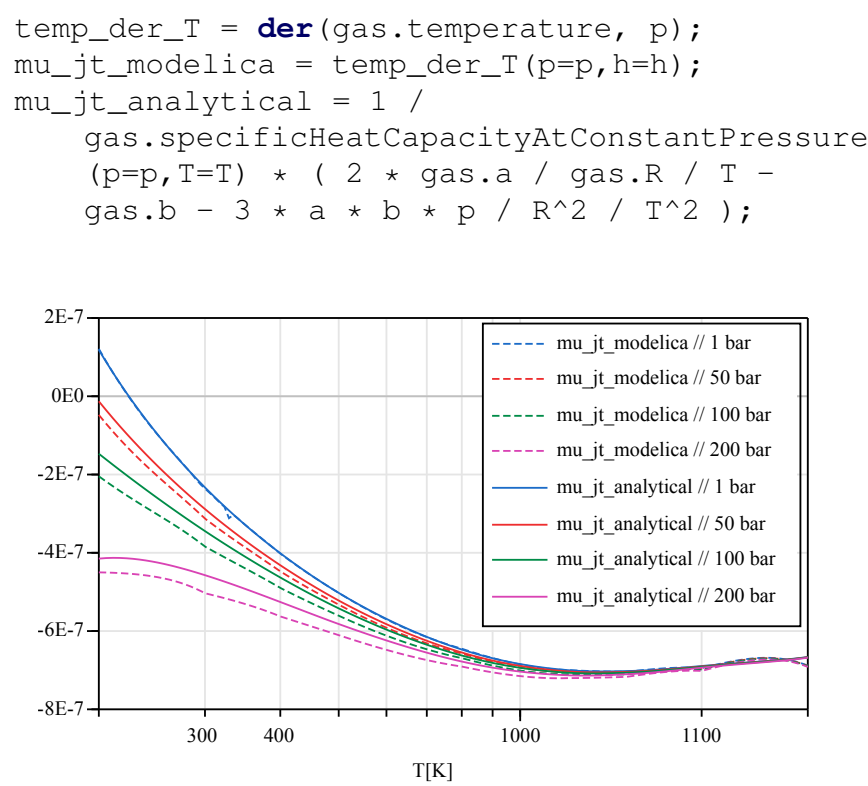

Figure 5. Analytical and Numerical Joule-Thomson coefficient for hydrogen.

The result for different pressures over the logarithmic scaled temperature is shown in Figure 5. For ambient pressure both definitions show the same behaviour. For higher pressures the assumption $v>>b$ from equation (27) cannot be met anymore and the analytical description of $\mu_{J T}$ recedes from the numerical derivative formulated in the $P S L$.

\section{Applications}

\subsection{Hydrogen fuel tank}

To simulate the filling process of a hydrogen tank a simplified model utilizing the Hydrogen Library as in Figure 6 is used. The fluid part consists of a pressure source at $200 \mathrm{bar}$, a variable orifice and a reservoir with a heat connection to the environment. The mass flow rate should be kept constant over the filling process, so a PI controller is used to set the valve opening. To restrict the maximum temperature in the tank during the filling process, the hydrogen gas from the pressure source is assumed to be precooled at $-10^{\circ} \mathrm{C}$.

\subsection{Results}

The temperature in the tank during filling process is plotted in Figure 7 for different hydrogen media models. The ideal gas model

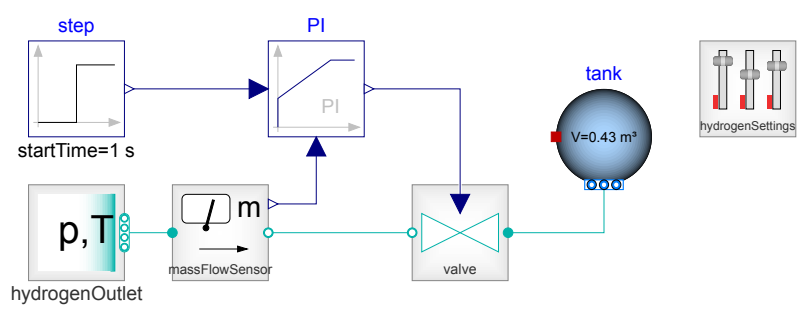

Figure 6. Dymola model to simulate a tank filling process for hydrogen.

(Modelica.Media.IdealGases.SingleGases.H2) strongly underestimates the maximum temperature as it only takes into account the temperature rise due to translational work from the pressure step at the inlet of the tank. Both the CoolProp and the van der Waals Real Gas Model consider the additional temperature rise due to the Joule-Thomson effect, while it is slightly overestimated by the van der Waals Real Gas Model. In the use-case of the tank filling process the safer approach is to use a model overestimating the temperatures, so the safety margin to the temperature limitation is increased.

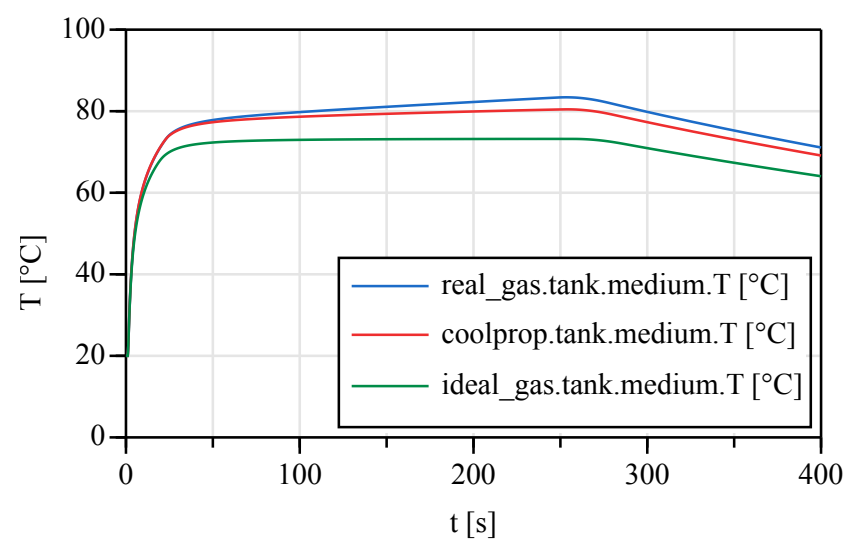

Figure 7. Results of the tank filling process simulation.

\subsection{Performance}

To compare the performance of the van der Waals gas model, the fuel tank experiment has been conducted using the Hydrogen Library ideal and van der Waals gas model as well as the CoolProp Library with the ExternalMedia interface (Casella and Richter, 2008). The results are summarized in table 2 .

Table 2. Results of the performance test.

\begin{tabular}{ll}
\hline Fluid model & Simulation time \\
\hline Ideal gas model / Hydrogen & $0.2 \mathrm{~s}$ \\
van der Waals model / Hydrogen & $1.06 \mathrm{~s}$ \\
CoolProp lib. / ExternalMedia & $4.18 \mathrm{~s}$ \\
\hline
\end{tabular}


The van der Waals model has a significant performance advantage against the ExternalMedia approach at the cost of lower accuracy.

\subsection{Excursus: Experiment of Gay-Lussac}

The experiment of Gay-Lussac was conducted in 1807 and repeated by (Joule, 1845). A volume with a high pressure at $22 \mathrm{bar}$ and environment temperature of $20^{\circ} \mathrm{C}$ is connected to an evacuated volume and the gas flows until both pressures are equal. After reaching thermodynamic equilibrium, the same temperature as the start temperature was measured in both volumes. With the measurement accuracy of that time the ideal gas law was proven. The model shown in figure 8 is an example model of the PSL. The two volumes are modeled as spheric tanks with a heat transfer connection. Between the pneumatic ports of the volumes a V22 valve prevents air flow. After one second the valve opens and gas flows until the equilibrium is reached.

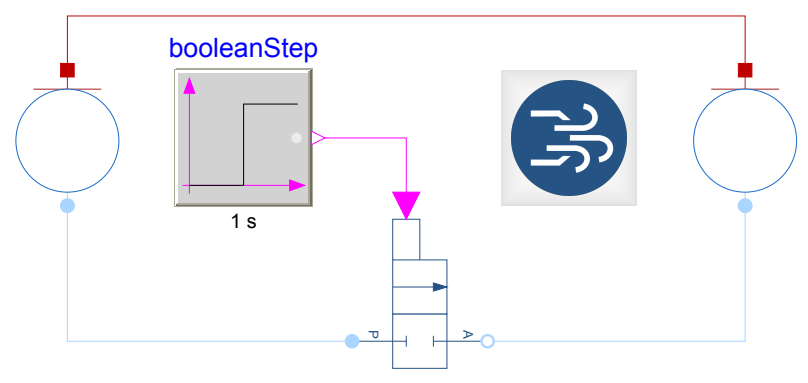

Figure 8. Dymola model to simulate the experiment of GayLussac.

With more accurate measurement it was found that the temperature after reaching thermodynamic equilibrium was $3 \mathrm{~K}$ below the initial temperature. As the experiment did not allow any change in enthalpy, this behavior could only be explained by the real gas law. The results in table 3 show that the van der Waals model covers this real gas effect.

Table 3. Results of the experiment of Gay-Lussac.

\begin{tabular}{ll}
\hline Fluid model & End temperature \\
\hline Ideal gas model / PSL & $20.0^{\circ} \mathrm{C}$ \\
van der Waals model / PSL & $17.2^{\circ} \mathrm{C}$ \\
CoolProp lib. / ExternalMedia & $16.9^{\circ} \mathrm{C}$ \\
\hline
\end{tabular}

\section{Summary}

The investigation shows that the van der Waals Real Gas Model is a good compromise between accuracy and simulation performance. A big advantage of the model is the possibility to describe the influence of the internal pressure on the specific enthalpy fully analytical. Thus it can be reduced to a single additional term in the formulation of the internal energy. A van der Waals model can be easily adapted to different gases as the formulation only requires pressure and temperature at the critical state, the molar mass and a table for the temperature-dependent part of the internal energy.

\section{References}

C. J. Adkins. Equilibrium Thermodynamics (3rd ed.). Cambridge University Press, Cambridge, UK, 1983. ISBN 0-52125445-0.

Ian H. Bell, Jorrit Wronski, Sylvain Quoilin, and Vincent Lemort. Pure and pseudo-pure fluid thermophysical property evaluation and the open-source thermophysical property library coolprop. Industrial \& Engineering Chemistry Research, 53(6):2498-2508, 2014. doi:10.1021/ie4033999. URL http://pubs.acs.org/doi/abs/10.1021/ ie4033999.

H. B. Callen. Thermodynamics and an Introduction to Thermostatistics. John Wiley \& Sons, New York, 1985. ISBN 978-0471862567.

Francesco Casella and Christoph Richter. Externalmedia: A library for easy re-use of external fluid property code in modelica. Proceedings of 6th International Modelica Conference, pages 157-161, 2008.

J. D. Van der Waals. The equation of state for gases and liquids. Nobel Lectures, Physics 1901-1921, pages 254-265, 1967.

Walter Greiner, Ludwig Neise, and Horst Stöcker. Thermodynamik und statistische Mechanik. Verlag Harri Deutsch, Frankfurt am Main, 1993. ISBN 978-3808557082.

J.P. Joule. Liv. on the changes of temperature produced by the rarefaction and condensation of air. The London, Edinburgh, and Dublin Philosophical Magazine and Journal of Science, 26(174):369-383, 1845. doi:10.1080/14786444508645153.

Eric W. Lemmon, M. L. Huber, and M. O. McLinden. NIST Standard Reference Database 23: Reference Fluid Thermodynamic and Transport Properties - REFPROP. National Institute of Standards and Technology, Standard Reference Data Program, Gaithersburg, 9.0 edition, 2010. URL http: //www.nist.gov/srd/nist23.cfm.

W. Moore. Grundlagen der Physikalischen Chemie. De Gruyter, Berlin, 1986. ISBN 978-3110099416.

W. Sutherland. The viscosity of gases and molecular force. Philosophical Magazine 5, pages 507-531, 1893.

N. W. Tschoegl. Fundamentals of Equilibrium and Steady-State Thermodynamics. Elsevier, Amsterdam, 1983. ISBN 0-44450426-5.

Office of energy efficiency US DOE, Renewable energy, the FreedomCAR, and fuel Partnership. Targets for onboard hydrogen storage systems for light-duty vehicles. Technical report, 2009.

B. Weigand, J. Köhler, and J. v. Wolfersdorf. Thermodynamik kompakt. Springer-Verlag, Berlin, 2008. ISBN 978-3-54071865-9. 